\title{
Classifying Best Access Points for Return of External Flows into Flowsheets
}

\author{
Pejman Oghazi ${ }^{1, *}$, Bertil Pålsson ${ }^{1}$ and Kent Tano ${ }^{2}$ \\ ${ }^{1}$ Luleå University of Technology, SE-971 87 Luleå, Sweden \\ ${ }^{2}$ LKAB, SE-983 32 Malmberget, Sweden
}

\begin{abstract}
External flows are process streams that come from auxiliary processes or events. They are re-routed into the ordinary flowsheet since they are thought to be too valuable to be sent to any tailings pond. External flows come from multiple sources, e.g. drainage sumps, spillage thickeners, depleted products etc. Therefore, external flows may fit or not fit into an existing flowsheet depending on several factors like, flow rate frequency, dilution ratio variation, chemical and mineralogical composition, particle size or particle morphology.

By using Particle Texture Analysis to investigate external flows and compare them with existing ordinary flows it is possible to pinpoint from a process mineralogy via point to what extent the external flow fits into the ordinary processing flowsheet. Results from this information category helps to reach a higher quality of process knowledge and control for every step in the concentrator.

Results show that some recycled flows reconnected to the main flow are not connected to the best point. A side effectof the analysis is that some flows may be sent to later grinding stages. Thus, decreasing the load on the primary mill, and increasing the retention time.
\end{abstract}

Keywords: Process optimisation, mineral processing, process mineralogy, recycled flows, Particle texture analysis.

\section{INTRODUCTION}

Selection of the best process for a plant is a vital task for a process engineer. In doing so it is important to have full knowledge of the variables that affect decisions such as mineralogical and process data or simply to know what sort of material flows into the plant. In a processing plant there are numerous processes and flows that are connected to each other so that a minimum of the valuable component that is being processed goes to waste. What is often the case in mineral processing is that flows are lumped together and it is important to check when this is the best solution for the process. In this context external flows are those that come from auxiliary processes and are re-routed into the ordinary flowsheet. Detailed knowledge of these flows moving around in the flowsheet is often missing.

The purpose of this study is to try to understand the difference between the external flows that are connected back to the ordinary flow. It is important to know what is sent back to the main flow. An investigation of the material that comes into the plant is important to reduce irregularity in the process. The topics are formulated into two research questions:

- What are the specific characterization methods that can be used to classify different flows?

\footnotetext{
*Address correspondence to this author at the Division of Mineral Processing, Luleå University of Technology, SE-971 87 Luleå, Sweden;

Tel: +46 920 491692; Mob: +46736878623; Fax: +46920 97364;

E-mail: Pejman.Oghazi@ltu.se
}

- Is it possible to pinpoint any decisive mineralogical factor for re-routed flows that are to be connected to the main process flow?

The study was carried out at the LKAB (LuossavaaraKiirunavaara AB) Malmberget concentrator. In the concentrator, ball mill grinding is used consecutive in three following steps and with wet low intensity magnetic separators in between. It is important to grind to, approximate $68 \%$ < $45 \mu \mathrm{m}$ to liberate gangue minerals, and to reach the desirable size distribution for the pellets feed. For more details of the process cf. [1].

\section{THEORETICAL FRAMEWORK}

The ore is coming from different ore bodies with and behave. This means that there could be a risk for quality variation in the final product, this is why traceability become a important aspect so the mineralogical description does not differ when the raw materials are mixed. By collecting information and knowledge from the flows, they can be operated to the adjusted part of the process.

Firstly it is imperative to describe the mineralogy of the material that is coming into the concentrator. The iron ore minerals are both magnetite and hematite, but (in Swedish operations) magnetite is the more common of the two. The important gangue minerals of the ores are: apatite and feldspar (microcline) since they are quality issues for the produced iron ore pellets. (can quantitative information be included?) Furthermore, the final circuit concentrate should have a total gangue content $<1.5 \%$. 
Secondly, to understand the fundamentals of the mineral processing it is important to understand and link every single stage in the process chain.(how can this be achieved?) It is the mineralogy and the properties of an ore that determine the conditions for the further processing. To meet these challenges an efficient process can be designed and the mineral treatment may be optimized [2]. Process mineralogy is a useful tool for flowsheet development and selection of the optimal process for the specific plant (reference). Process mineralogy explains the characterization of minerals according to how they behave during specific treatment, and gives us useful information such as grain size, mineral association, liberation and trace element content that affect the process [3].

All mineral companies struggle to have as high recovery as possible and to do so information that characterizes the minerals is vital. In the present case, Particle Texture Analysis (PTA) is used for getting information on the process mineralogy. By using Back Scattered Electron (BSE) from the scanning electron microscopy, images are analyzed by means of grey levels and every grain of interest is analyzed by X-rays. All analysed grain size fractions are imported to the PTA software, there images analyses are done offline to process and evaluate if grains occur as liberated or in composite particles [4].

\section{EXPERIMENTAL}

It is important to realise that the raw material for the concentrator comes from several ore bodies that are mixed in the process and this means there will be ores having different Fe-content and levels of contaminants [5]. The ores are treated in three major parallel grinding sections cf. [6]. All the spillage from the concentrator is reprocess in section of pre-concentration again (Fig. 1.)

Samples from nine different points were taken in the concentrator's section for external flows, cf. Fig. (1). Most samples are from flows that are to be recycled into the main process flow from thickeners and magnetic separators. In Table 1 all the sample points are presented. Sample points 18 are chosen to cover all the recycled material types that are going back to the main stream. Short explanation can be found in Table 1 below. Different amount of material were

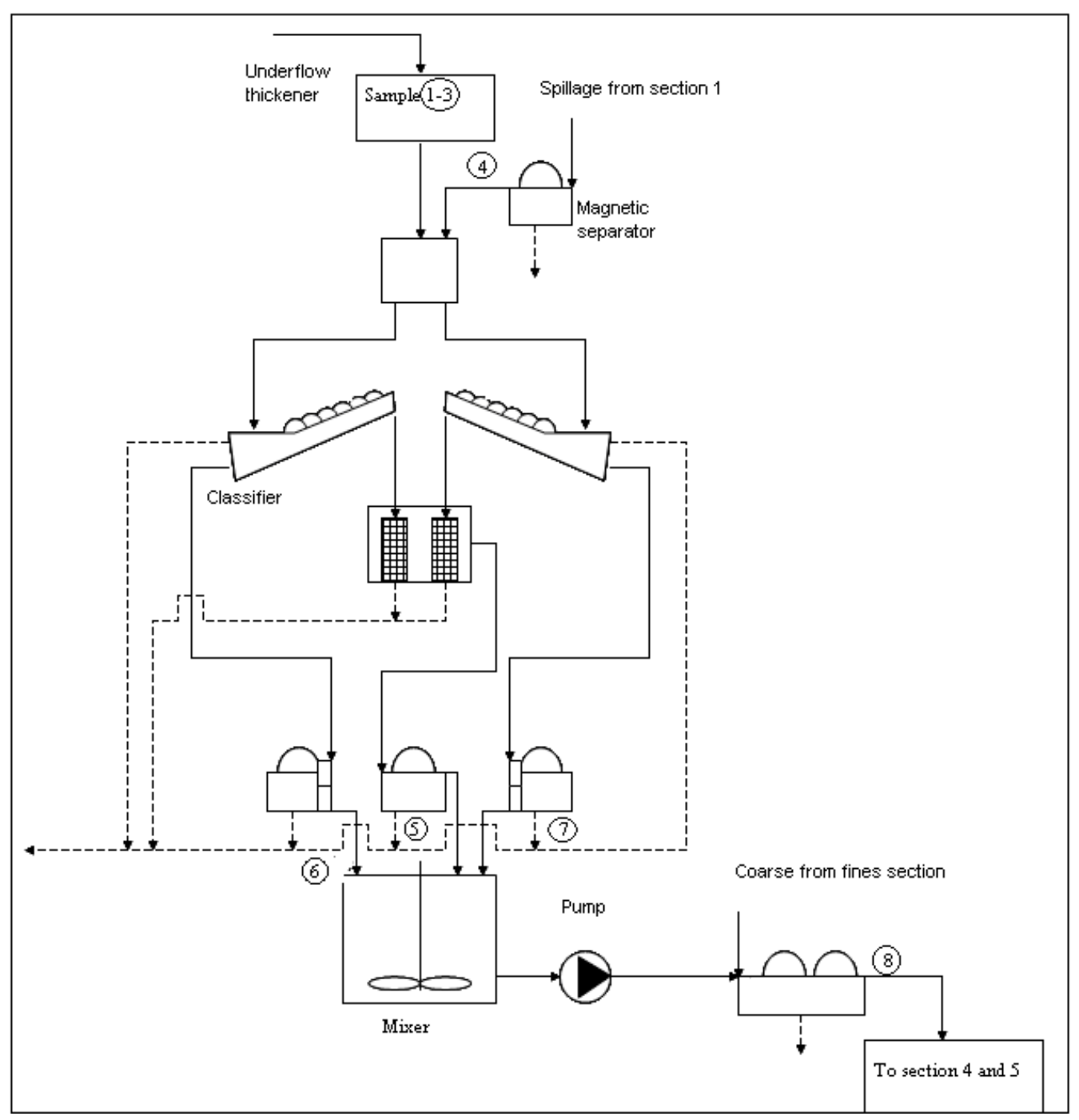

Fig. (1). Scheme over sample points in the section for pre-concentration and dewatering of external flows. Sample point \#9 is located in main grinding sections. The recycled flow is approximately $10 \%$ on a tonnage basis compared with the fresh flow to the concentrator. 
taken from the bulk and it is important that the samples are representative. Samples from points 1 to 9 were taken manually from the dressing plant. They were taken before in 20 minutes interval. Sample point 9 is a reference sample from the final magnetic separator in section 6 that gives information on the material that leaves the concentrator.

All the samples were dried and cut by a Jones splitter into suitable proportions in the laboratory at Luleå University of Technology (LTU). The dry material was sieved with a RoTap shaker down to $75 \mu \mathrm{m}$ and wet sieved further to $38 \mu \mathrm{m}$. Samples $38-53 \mu \mathrm{m}$ were sent to NTNU, Norway for particle texture analysis.

\section{RESULT}

The modal mineralogy from Fig. (2) shows that in the underflow from thickener number two (sample \#1) there is mostly magnetite while in thickeners four and five (sample $\# 2$ and \#3) there is around $25-30 \%$ magnetite and the rest is gangue minerals.

The material that is sent to thickeners four and five is the tailings from the wet cobbing separators that contain mostly gangue minerals. The modal mineralogy for samples \#2 and \#3 looks almost similar to each other, the reason is that the feed material for both thickeners is the same and is split between the thickeners. The $d_{80}$ is also approximately the same for both thickeners.

Sample \#4 (wet LIMS concentrate) in Fig. (3) shows the same result (high magnetite content) as sample \#1. Therefore, these two streams would be better sent directly to the tertiary mill in the main grinding section. It is unnecessary to grind them more because of their high content of magnetite, and they are also much finer compared to samples \# 2 and \#3.

Table 1. Sample Points with Explanation and Information

\begin{tabular}{|c|c|c|c|}
\hline Samples Point & Sample Explanation & $\mathbf{d}_{80}(\mu \mathrm{m})$ & $\%<45 \mu \mathrm{m}$ \\
\hline 1 & $\begin{array}{l}\text { Underflow thickener \# } 2 \\
\text { Sample contains a mix from section } 1-3 \text {, mostly hematite with a large variation. }\end{array}$ & $120 \mu \mathrm{m}$ & $22 \%$ \\
\hline 2 & $\begin{array}{l}\text { Underflow thickener \# } 4 \\
\text { Contains waste from wet cobbing plant section 4-5, also tails from magnetic separators section } 3 \text { and section } 6 \text {. }\end{array}$ & $210 \mu \mathrm{m}$ & $18 \%$ \\
\hline 3 & $\begin{array}{l}\text { Underflow thickener \# } 5 \\
\text { Thickener \#5 parallel to \#4 }\end{array}$ & $210 \mu \mathrm{m}$ & $22 \%$ \\
\hline 4 & Concentrate from magnetic separator. Feed is spillage from secondary mill section 1 . & $110 \mu \mathrm{m}$ & $11 \%$ \\
\hline 5 & Concentrate from magnetic separator. Coarse particles from classifier. & $380 \mu \mathrm{m}$ & $14 \%$ \\
\hline 6 & Concentrate from magnetic separator. Fine particles from classifier. & $111 \mu \mathrm{m}$ & $32 \%$ \\
\hline 7 & Concentrate from magnetic separator. Fine particles from classifier. & $105 \mu \mathrm{m}$ & $37 \%$ \\
\hline 8 & Concentrate from magnetic separator, feed is combined flows material collected from the re-routed flows. & $211 \mu \mathrm{m}$ & $22 \%$ \\
\hline 9 & Concentrate from magnetic separator, from tertiary mill in grinding section 6 . Used as reference. & $75 \mu \mathrm{m}$ & $23 \%$ \\
\hline Feed Mill \#1 section 5 & Primary mill feed. Used as reference. & $4780 \mu \mathrm{m}$ & $3 \%$ \\
\hline
\end{tabular}

Modal mineralogy

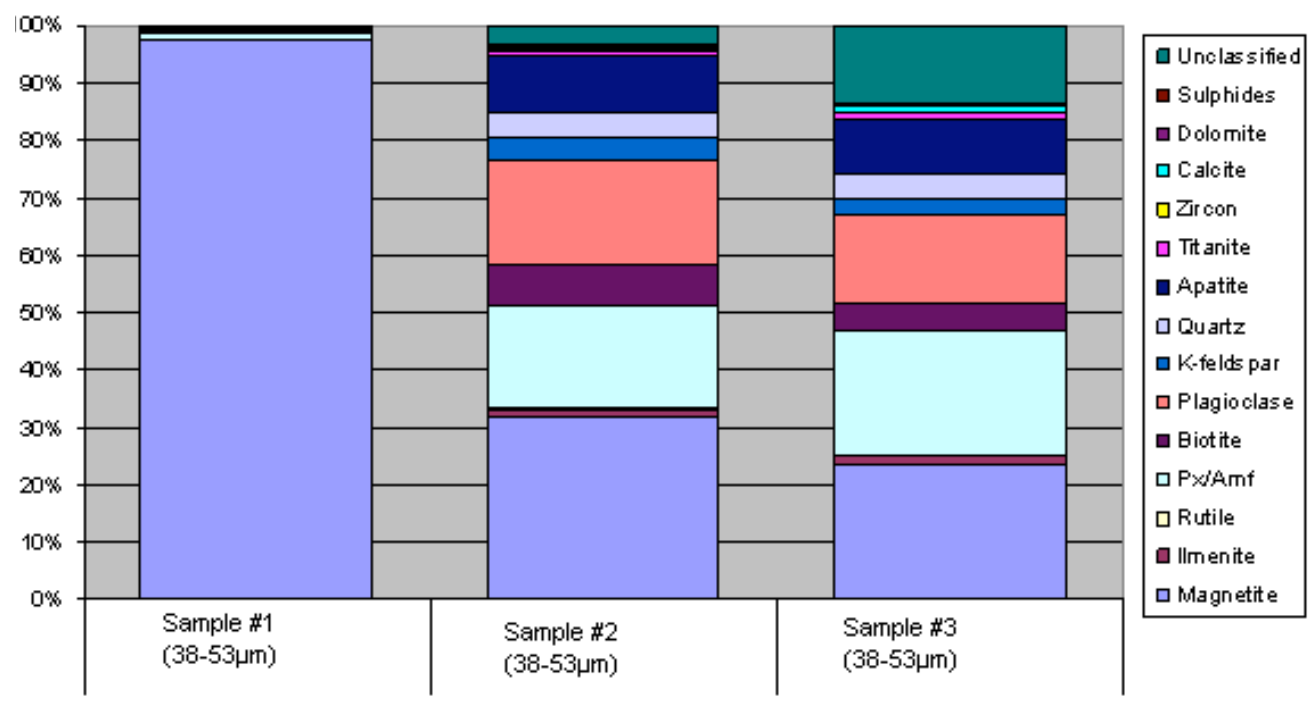

Fig. (2). Modal mineralogy from samples 1-3, taken from thickener underflows. 


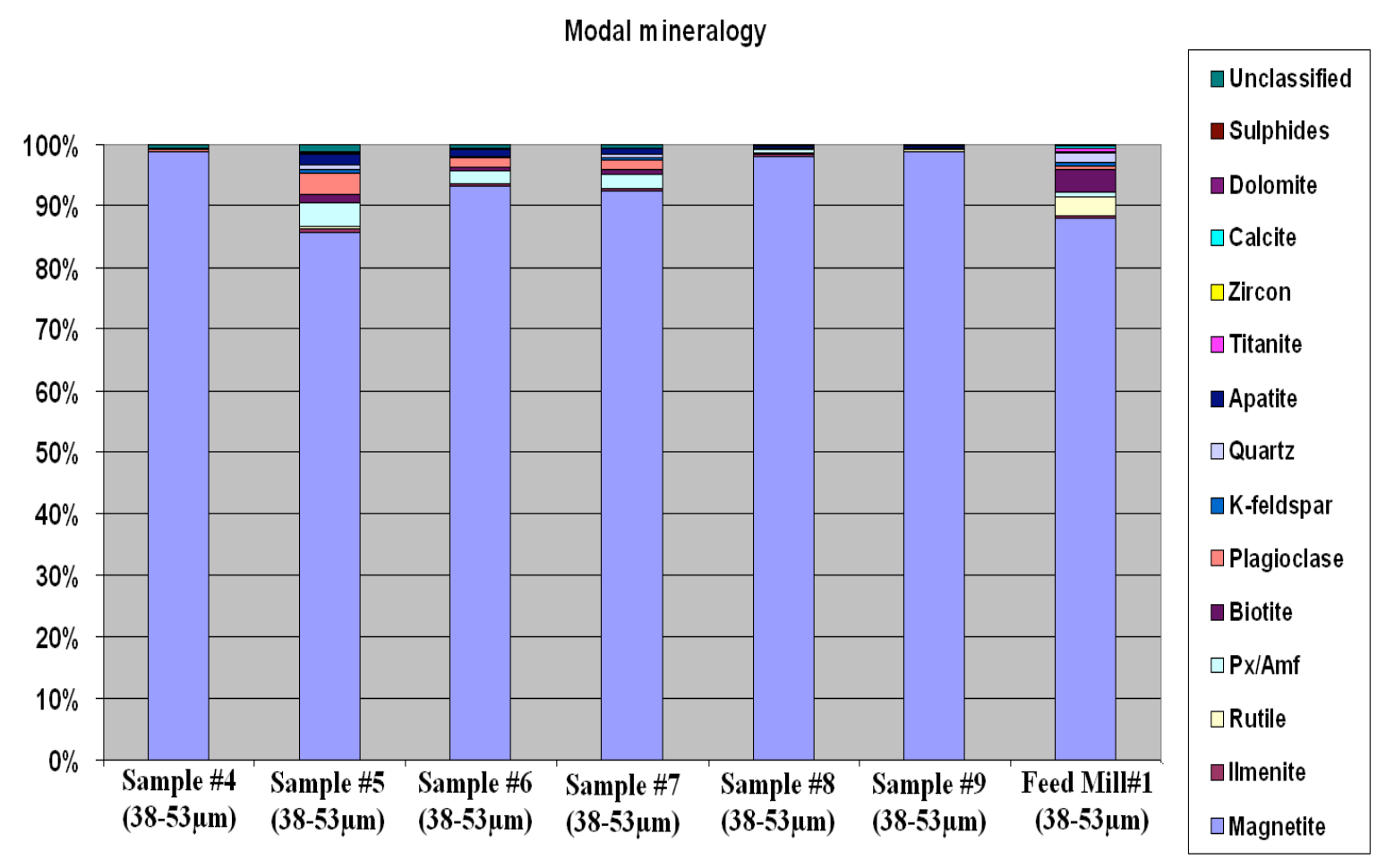

Fig. (3). Modal mineralogy from samples taken from magnetic separators and a primary mill in the concentrator.

Fig. (3) shows the modal mineralogy from the concentrates of magnetic separators that are used in the recirculation circuit except for sample \#9 which is taken from the final magnetic separator after the tertiary grinding stage. Sample \#9 is the reference here, representing the desired product that all other samples are compared with. The concentrate from the concentrator (in this case sample \#9) goes to the next process step, which is the pelletising plant. As seen in Fig. (3), the gangue content in sample \#5 is almost double compared to sample \#6 or \#7 while the last mentioned samples are almost identical. Materials in sample \#5 are the coarse discharge from two spiral classifiers. The overflow from the same spiral classifiers that contains fine particles and enters the magnetic separators represented by samples \#6 and \#7. In Table 1 can be read that the $d_{80}$ for sample \#5 is much coarser compared to sample \#6 and \#7 and should so be. Fig. (3) illustrate that there are more gangue minerals in the coarse stream from the classifiers which is logical.

All recycled flows go through a magnetic separator (sample \#8) and from this the material is pumped to the primary mill in section 5. By comparing the existing feed entering the primary mill with the material in sample \#8 that is sent to the primary mill it shows that sample \#8 contains over $96 \%$ magnetite and a particle size distribution with a $\mathrm{d}_{80}$ around $200 \mu \mathrm{m}$, but the feed to the primary mill has $86 \%$ magnetite and $\mathrm{a} \mathrm{d}_{80}$ is around $4800 \mu \mathrm{m}$. Therefore, sample \#8 does not match with the feed to the primary mill. To obtain a better process the concentrate (sample \#8) should bypass the primary mill and preferably be sent to the secondary mill since the material properties are similar. Today's reason for not sending it to the tertiary mill is that there is one coarse intermittent flow entering before sample point \#8. Could this coarse flow be treated separately sample \#8 might be sent directly to the secondary mill.
Today this material (sample \#8) is sent to the primary mill, but it is too fine for the primary mill. It will only create an unnecessary volumetric load and reduce the retention time in the primary mill. The modal mineralogy in Fig. (3) shows that there is mostly magnetite in sample \#9 (final concentrate); however, it also contains a few particles of gangue minerals. One of the advantages with modal mineralogy is the possibility to exactly know how much of a certain mineral that exists in the sample. Counting the particles in the final concentrate gives that of totally 4513 particles there were 31 gangue mineral particles and the rest of it was magnetite. Thus, the material that leaves the concentrator contains 99 percent magnetite.

Fig. (4) illustrates the mineral associations to quartz and feldspar in the thickener underflows. Most of the quartz and the feldspar are liberated. However, around 10 percent of them are associated to magnetite. This means that these mixed particles need some regrinding, otherwise they will increase the $\mathrm{SiO}_{2}$ content of the final pellets.

The association of minerals to apatite is shown in Fig. (5). Sample \#1 was shown earlier (Fig. 2) to contain mostly magnetite, but there were small portions of other minerals as well. Fig. (5) demonstrates that none of the magnetite in sample \#1 is associated to apatite.

The similarities between sample \#1 and sample \#4 are shown once again in Fig. (5 and 6). This result further substantiates the recommendation to bypass the primary mill and send these streams to the secondary or tertiary mill in the grinding section. For samples \#4-\#9 there were not much quartz and feldspar associations in the samples. Therefore, the analyses are not presented in this paper. However, there were some $(4 \%)$ in sample \#8, but that most likely comes 
Minerals associated to Quartz and feldspar

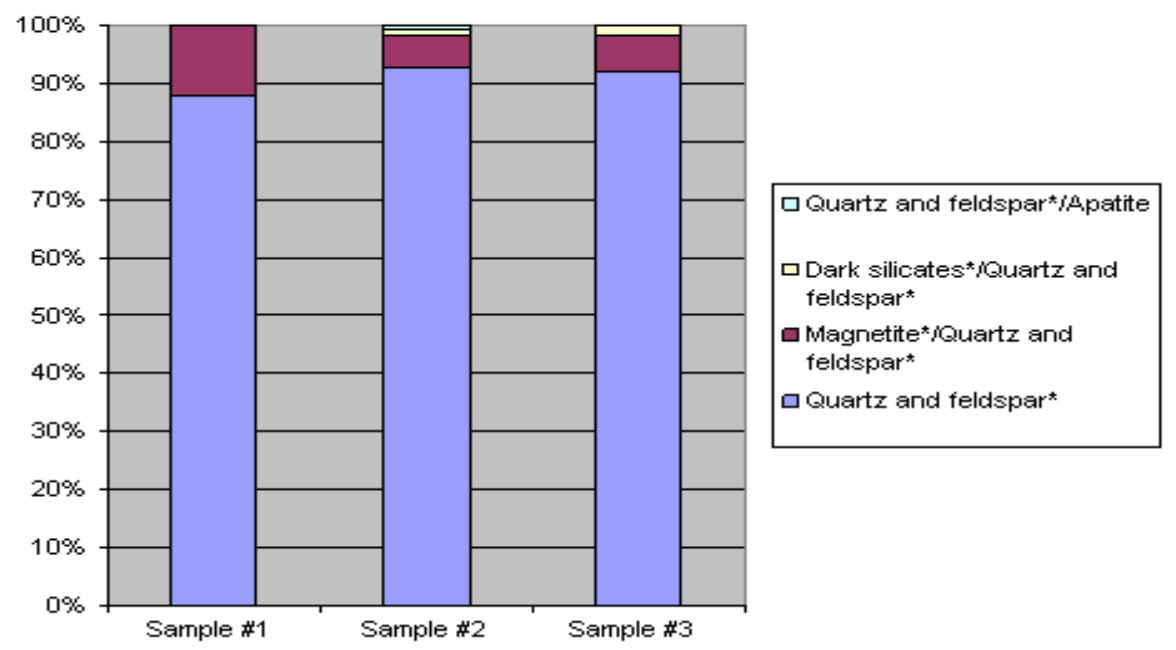

Fig. (4). Mineral association to Quartz and Feldspar in thickener underflows.

Minerals Associated to Apatite

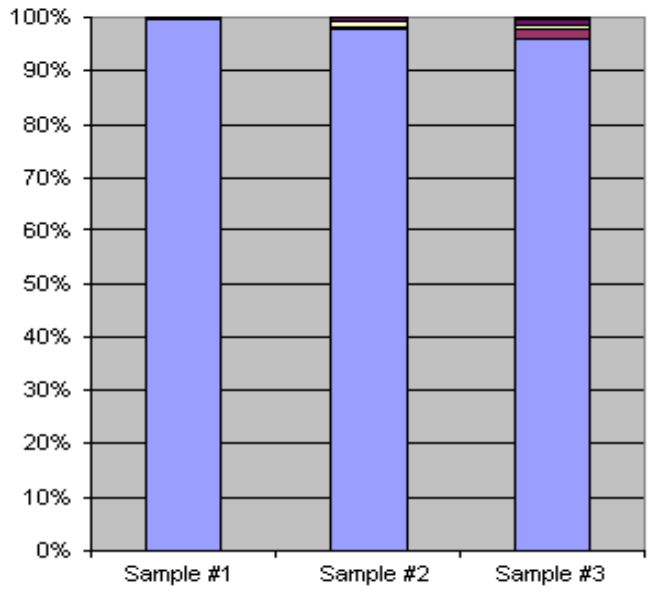

\begin{tabular}{l}
\hline Apatite \\
Quartz and \\
feldspar /Apatite \\
$\square$ Magnetite Dark \\
silicates*/Apatite \\
QDark \\
silicates /Apatite
\end{tabular}

Fig. (5). Mineral association to Apatite in thickeners

\section{Minerals associated to apatite}

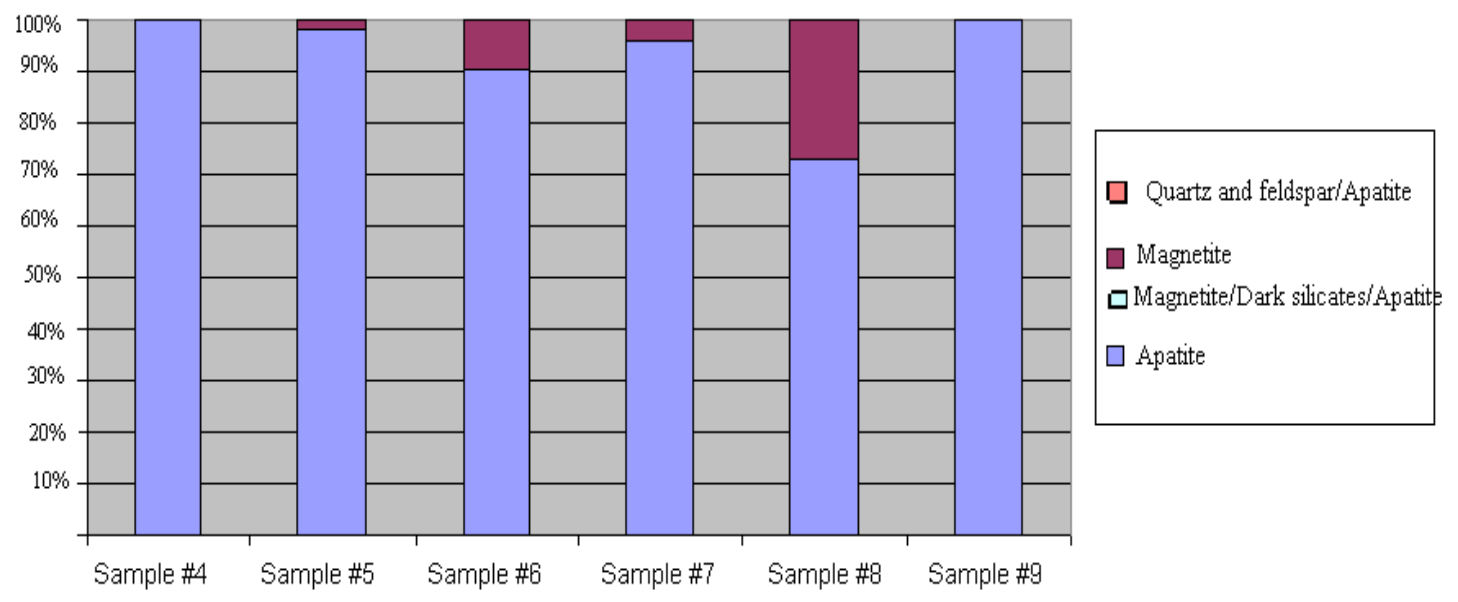

Fig. (6). Mineral association to Apatite in concentrate from magnetic separators. 
from samples \#2 and \#3 (underflow thickeners) or the coarse intermittent flow entering just before point \#8.

Analysis from samples \#5-\#8 shows that there are some apatites mainly associated to magnetite. On the other hand for sample \#8 it is clear that over $25 \%$ of the apatite is associated to magnetite and this is particularly high compared to other samples from the recirculation section. It can be explained by the coarse intermittent particle stream which enters the magnetic separator at this position. There were few apatite grains in sample $\# 9$, which is the final concentrate.

\section{CONCLUSIONS AND DISCUSSION}

Modal mineralogy is one way to present the results from the process mineralogy analyses. It is a convenient way to understand the content of different flows. This type of analysis may be used to:

- Identify what sort of material is pumped to the grinding section

- Optimise the process by sending external flows to the best access point in the main flowsheet

- Reduce any overloading in any process step by decreasing unnecessary volumetric flows

This way for collecting total information on how each unit operation is working and what sort of material is recycled to the main flowsheet is very effective. The ideal case is when complete information for each external flow that is added to the main streams exists and it is then joined to an optimal position in the main flowsheet. In this case circulating flows are today collected in a mixer, and later going throw a magnetic separator before they are sent to the primary mill. As mention before underflow thickener \# 2 or sample \#1 is not returned to the best process point and it puts an unnecessary load on some stages in the main flowsheet. The result from modal mineralogy shows that there were some gangue minerals in the streams from the thickeners and also some in intermediate streams. Most of them are gone after the re-treatment section. Based on gangue content and particle size only this combined flow should bypass the primary mill. However, if the rapidity of the analysis was better maybe this method would be more common in the daily process.
Minerals association analyses on these samples are a more informative and important way to pinpoint how nonliberated gangue minerals occur in these streams. If the gangue minerals are locked with magnetite they will ultimately end up in the final concentrate since current lowintensity magnetic separators pick any particles with small magnetic volumes. Therefore, mineral association analysis is the most important tool in deciding where to send recycled flows. However, to avoid data overload it is better to first use particle size and modal mineralogy data to make a first selection of possible connection points. In a second step the mineral association may be used to confirm the selection or to do an incremental change.

\section{FUTURE WORK}

The next step in the development work will be to use the data from the PTA and investigate it with Multivariate Data Analysis to see in detail to what degree the particle morphology is affecting the process. Even here, a comparison of main stream flows with external flows to check for relationships will be done, but with the added benefit of an unbiased analysis.

\section{ACKNOWLEDGEMENTS}

The authors are grateful for the financial support by LKAB. They are also grateful for the assistance during sampling at LKAB, Malmberget.

\section{REFERENCES}

[1] B. Kvarnström, and P. Oghazi, "Methods for traceability in continuous processes-Experience from an iron ore refinement process", Miner. Eng., vol. 21, no. 10, pp. 720-730, 2008.

[2] D. N. Sutherland, and P. Gottlieb, "Application of automated quantitative mineralogy in mineral processing", Miner. Eng., vol. 4, pp. 753-762, 1991.

[3] J. Zhou, Y. Gu, and H. Y. Zhou, "Process mineralogy and applications in mineral processing”, In: Proceeding of XXIV International mineral processing congress, Beijing China, 2008, pp. 107-117.

[4] K. Moen, Quantitative measurements of mineral microstructure, Doctoral. Thesis, Department of Geology and Mineral Resources Engineering, Trondheim, Norwegian University of Science and Technology, Norway, 2006, pp. 1-120.

[5] O. Martinsson, and C. Wanhainen, "Excursion Guide, GEODE work shop" Retrieved 1 October, 2007. [Online]. Available: http://www.gl.rhbnc.ac.uk/geode/KirunaGuide. [Accessed 2007].

[6] K. Tano, B. Pålsson, J. Alatalo, and Lindqvist, "The use of process simulation methodology in process design where time and performance is critical", SME 2006. 